\title{
Fuzzy Logic Expert System for Optimum Maintenance of Power Transformers
}

\author{
Chilaka Ranga ${ }^{1}$, Ashwani Kumar Chandel $^{2}$ and Rajeevan Chandel $^{3}$ \\ ${ }^{1,2}$ Department of Electrical Engineering, \\ ${ }^{3}$ Department of Electronics and Communication Engineering \\ National Institute of Technology Hamirpur, Himachal Pradesh, 177005, India
}

\begin{abstract}
Condition monitoring of power transformers improves the reliability and the safety of an electrical power network. It protects the transformers from failures, and avoids huge revenue loss to utilities and customers. The health status of transformers is decided by several influencing factors. An accurate health assessment of transformers has been a challenging task for the researchers as well as the transformer diagnostic experts. In the present paper, a new fuzzy logic model is proposed to assess the impact of all such factors on the overall health index of the transformers, and in turn their health status. It incorporates the information obtained from various attributes which have been categorized into four different groups namely, transformers tests and measurements, visual inspection, maintenance and operation history, and design and fabrication. These attributes are initially assigned appropriate scores based on their present status/condition. Further, the proposed fuzzy logic model is developed based on the total scores attained by the four design categories. The present analysis avoids the over impact of the attributes thereby resulting in a more reliable and accurate health index of transformers. It overcomes the shortcomings of the conventional health assessment methods of transformers.
\end{abstract}

Keywords: Transformer, attribute, fuzzy logic, health index, diagnosis.

\section{Introduction}

Power transformers are the most significant devices in substations, and play an important role in electricity transmission. Abnormalities if present in transformers accelerate their aging process. It may finally lead to failure of transformers resulting in a big revenue loss not only to utilities but also to customers [1,2]. Therefore, the health assessment of transformers has gained an immense importance in recent years [3]. Very often it is observed from the literature that the transformers health condition has been mainly assessed based on the information extracted from solid and liquid insulations [4]. Aging of these insulations accumulates various gasses into the transformer oil. The concentrations of these gasses are normally measured by the dissolved gas analysis (DGA) which is a prominent diagnostic test for the transformers. The concentrations of these dissolved gasses have been further utilized to determine the health condition as well as the type of incipient fault present in transformers [5]. Several conventional methods such as Key gas [6], Rogers ratios [7], [8], Duval Triangle [9], [10], Dornenburg ratios [11], [12], modified Rogers ratios [10], [11] and IEC/IEEE ratio code methods [12], [13] have been developed and reported in literature for an interpretation of these dissolved gasses. Also the other tests such as break down voltage (BDV), water content, degree of polymerization (DP) and furan content (FC) of solid insulation, partial discharge (PD), tensile strength (TS), interfacial tension (IFT), flash point (FP), bushing tests, tap changer tests etc. have been utilized to assess the present health status of the transformers [14-16]. Each of these tests has its own importance in determining the health of the transformers. During the past few years, various fuzzy logic models have been developed based on one or fewer of these diagnostic tests, to determine the overall health index of the transformers [17-20]. Fuzzy logic is a very helpful tool to determine the health index of power transformers [18]. It is a logical system that provides a very convenient method to map the input to the output through linguistic rules formed from human understanding rather than stringent mathematical models [17]. It can

Received: September $1^{\text {st }}, 2016$. Accepted: December $23^{\text {rd }}, 2016$ 
integrate the tests data of the transformers and the experience of transformer diagnosis experts.

It facilitates more reliable and accurate decision making of the health condition of transformers $[19,20]$. The power of fuzzy logic is most evident during the operation and inference stages of a process, deriving new results that then provide progressively more precise information about the actual condition of the equipment [19]. Moreover, fuzzy logic is useful for managing uncertain and vague information. Hence, fuzzy logic is an ideal tool for managing imprecise and vague information in the real world [18]. Health index quantifies the equipment condition based on long-term degradation factors thus cumulatively leading to an end of the life of the asset [17]. Although the fuzzy models developed earlier have their own importance in determining the overall health index of transformers, however none of them consider the impact of all factors which affect the health condition of the transformers $[18,19]$. An accurate health assessment of transformers not only depends on its insulation test results but also on several other factors corresponding to design and fabrication, maintenance and operating history, visual inspection etc. [20-22]. Consequently, there is a need for a new approach which assesses the impact of all influencing attributes of transformers. This task has been accomplished in the present paper.

In the present paper, a new fuzzy logic (FL) model is proposed to determine the overall health condition of transformers based on the data gathered from their several influencing factors. The attributes of transformers have been classified into four categories. Further each of the attributes has been subdivided into four groups namely, low, medium, high and very high. Thereby, a new fuzzy logic model is designed based on the total ranges obtained for each of the four categories. Twenty-five transformers operated by Himachal Pradesh State Electricity Board, India were tested and examined to prove the validity and efficiency of the proposed model. The proposed fuzzy model can enhance the performance and operational capability of the transformers.

\section{Transformer Attributes}

Multi-attributes analysis integrates the information obtained from several factors of transformers [23]. In this method, the various attributes of transformers have been categorized into four groups viz. tests and measurements $\left(A_{1}\right)$, visual inspection $\left(A_{2}\right)$, maintenance and operation history $\left(\mathrm{A}_{3}\right)$, and design and fabrication $\left(\mathrm{A}_{4}\right)$. According to these four categories, a total of thirty attributes given in Table 1 have been identified [24-26]. It is observed from literature that the health assessment of transformers mainly depends on several diagnostic tests and measurements. The significance and interpretations of these diagnostic tests can be found in $[24,25]$. A few of these tests and measurements are discussed in the subsequent paragraphs. Dissolved gas analysis is the most widely performed test to determine the health condition of the transformers, and to identify the incipient faults present within transformers. In this test, the total dissolved combustible gas (TDCG) concentration specifies the overall condition of transformer [11]. The maximum voltage that can be applied across the insulation without any electrical breakdown is called as dielectric strength of insulation [9]. Any significant reduction in the dielectric strength indicates that the oil is no longer capable of performing its vital function. The degree of polymerization and furan content test of transformers decide the health status of their solid insulation [19]. When a cellulose molecule breaks into smaller lengths or ring structures, a chemical compound known as a furan is formed. This is a measure of the insulating paper quality (mechanical strength) and hence an indicator of the consumed lifetime of a transformer [19].

Excessive water content in transformer insulation decreases its breakdown strength. Ultimately it results in the loss of insulation performance. The dissipation factor is a measure of the power lost in the transformer oil during the operation of transformer [17]. It increases as per the deterioration of the oil. Similarly, the total acidity of transformer oil is another indication for the deterioration of the overall insulation system of the transformer. The total acidity increases continuously with extended service periods of the transformer insulation, and can therefore be used to indicate the health of the insulation $[17,19]$. 
Table 1. Transformer attributes corresponding to tests and measurements, visual inspection, maintenance and operation history, and design and fabrication [24-27].

\begin{tabular}{|c|c|c|c|c|c|}
\hline $\begin{array}{c}\mathrm{Sr} \\
\text { No }\end{array}$ & Attribute & \begin{tabular}{|c|}
$\mathrm{Sr}$ \\
$\mathrm{No}$
\end{tabular} & Attribute & $\begin{array}{c}\mathrm{Sr} \\
\text { No }\end{array}$ & Attribute \\
\hline & $\begin{array}{l}\text { Tests and measurements } \\
\left(\mathrm{A}_{1}\right)\end{array}$ & & Visual inspection $\left(\mathrm{A}_{2}\right)$ & 6 & $\begin{array}{l}\text { Bushing ambient temperature } \\
\text { (in }{ }^{0} \mathrm{C} \text { ) }\end{array}$ \\
\hline \multirow[t]{5}{*}{1} & DGA-TGCG (in ppm) & 1 & Presence of dust and dirt & & $30-40$ \\
\hline & $0-720$ & & None & & $40-50$ \\
\hline & $720-1920$ & & Little & & $50-60$ \\
\hline & $1920-4630$ & & Medium & & $60-75$ \\
\hline & $4630-10000$ & & High & 7 & Average load (in \%) \\
\hline \multirow[t]{5}{*}{2} & Oil BDV (in kV) & 2 & Oil leakage & & $50-85$ \\
\hline & $75-40$ & & None & & $85-90$ \\
\hline & $40-35$ & & Little & & $90-95$ \\
\hline & $35-20$ & & Medium & & $95-100$ \\
\hline & $20-0$ & & High & 8 & Repairs occurred in core \\
\hline \multirow[t]{5}{*}{3} & DP value (in ppm) & 3 & $\begin{array}{l}\text { Partial discharge white } \\
\text { powder }\end{array}$ & & none \\
\hline & $1400-1000$ & & None & & once \\
\hline & $1000-500$ & & Low & & twice \\
\hline & $500-200$ & & Medium & & $>$ twice \\
\hline & $200-0$ & & High & 9 & Power factor \\
\hline \multirow[t]{5}{*}{4} & Water content (in ppm) & 4 & Appearance of oil & & unity p.f. \\
\hline & $0-15$ & & $\begin{array}{l}\text { pale yellow and clear } \\
(\mathrm{PYC})\end{array}$ & & $1.0-0.9$ \\
\hline & $15-20$ & & bright yellow (BY) & & $0.9-0.85$ \\
\hline & $20-25$ & & brown & & $0.85-0.75$ \\
\hline & $25-50$ & & Black & 10 & Ambient temperature (in ${ }^{\circ} \mathrm{C}$ ) \\
\hline \multirow[t]{5}{*}{5} & $\begin{array}{l}\text { Furan content (2-FAL } \\
\text { in ppm) }\end{array}$ & 5 & Core noise (in $\mathrm{dB}$ ) & & $20-50$ \\
\hline & $0-0.1$ & & $6.02-21.58$ & & $(20-15)$ or $(50-60)$ \\
\hline & $0.1-1.0$ & & $0-6.02$ & & $(15-10)$ or $(60-70)$ \\
\hline & $1.0-10$ & & $21.58-25$ & & $(10-0)$ or $(70-90)$ \\
\hline & 10 to 20 & & $25-35$ & 11 & Tank damages \\
\hline \multirow[t]{5}{*}{6} & IFT (in $\mathrm{mN} / \mathrm{m}$ ) & & $\begin{array}{l}\text { Maintenance and } \\
\text { operation history }\left(\mathrm{A}_{3}\right)\end{array}$ & & none \\
\hline & $0-15$ & 1 & Oil age (in years) & & low \\
\hline & $15-20$ & & $0-10$ & & medium \\
\hline & $20-25$ & & $10-25$ & & high \\
\hline & $25-50$ & & $25-45$ & & Design and fabrication $\left(\mathrm{A}_{4}\right)$ \\
\hline \multirow[t]{5}{*}{7} & Dissipation factor & & $45-75$ & 1 & Fire protection system \\
\hline & $0-0.1$ & 2 & Winding age (in years) & & automatic protection (AP) \\
\hline & $0.1-0.2$ & & $0-10$ & & manual and automatic (MAP) \\
\hline & $0.2-0.3$ & & $10-25$ & & manual protection only (MP) \\
\hline & $03-0.6$ & & $25-45$ & & detection only/ nothing (DO) \\
\hline \multirow[t]{5}{*}{8} & $\begin{array}{l}\text { Turns ratio (in } \% \\
\text { deviation) }\end{array}$ & & $45-75$ & 2 & Type of cooling \\
\hline & $0-0.1$ & 3 & Core age (in years) & & water cooling (WC) \\
\hline & $0.1-0.3$ & & $0-10$ & & $\begin{array}{l}\text { forced oil, forced air cooling } \\
\text { (FOFAC) }\end{array}$ \\
\hline & $0.3-0.5$ & & $10-25$ & & forced air cooled (FAC) \\
\hline & $0.5-1$ & & $25-45$ & & oil natural cooling (ONC) \\
\hline \multirow[t]{4}{*}{9} & $\begin{array}{l}\text { Insulation resistance (in } \\
\mathrm{M} \Omega \text { ) }\end{array}$ & & $45-75$ & 3 & Type of radiator \\
\hline & $5000-3000$ & 4 & Winding faults & & flange \\
\hline & $3000-2500$ & & None & & Tabular \\
\hline & $2500-2000$ & & Once & & Other \\
\hline
\end{tabular}




\begin{tabular}{|l|l|l|l|l|l|}
\hline & $2000-0$ & & Twice & & None \\
\hline 10 & Acidity (in mg KOH/g) & & $>$ twice & 4 & Bushing type \\
\hline & $0-0.03$ & 5 & Bushings faults & & $\begin{array}{l}\text { oil impregnated paper bushing } \\
\text { (OIPB) }\end{array}$ \\
\hline & $0.03-0.15$ & & None & & $\begin{array}{l}\text { epoxy resin impregnated } \\
\text { bushing (ERIB) }\end{array}$ \\
\hline $0.15-0.3$ & & Once & $\begin{array}{l}\text { resin impregnated bushing } \\
\text { (RIB) }\end{array}$ \\
\hline & $0.3-0.5$ & & Twice & & resin bounded bushing (RBB) \\
\hline & & & $>$ twice & & \\
\hline
\end{tabular}

In addition to these factors of transformers, there are some other significant exterior factors which belong to visual inspection, maintenance and operation history, and design and fabrication [24]. Visual inspection of the transformer exterior reveals important perceptible data. Oil leaks, inoperative gauges, presence of dust and dirt, cracked bushings and oil colour are examined as per the details given in Table $1[25,26]$. The maintenance and operation history of transformers provide the information regarding the major failures and repairs of each component of the transformer [25]. Similarly, the information regarding the design specifications of each component is provided by the manufacturer [27]. At times it is observed that the effective performance of transformers is decided by the type of radiator, cooling, bushing and fire protection system, detailed in Table 1.

In the proposed multi-attribute analysis, each of the attributes corresponding to $A_{1}, A_{2}, A_{3}$ and $\mathrm{A}_{4}$ has been divided into four groups ( $\mathrm{i}=1$ to 4 ) namely, low, medium, high and very high (VHigh). The ranges or conditions for these four groups of every attribute were given in Table 1. Subsequently, the four groups of each attribute have been assigned appropriate scores or weights $(w)$ between 0 and 10 . The minimum and maximum limits of the weights for these four groups are $0 \leq w \leq 2.5(i=1), 2.5<w \leq 5 \quad(i=2), 5<w \leq 7.5 \quad(i=3)$ and $7.5<w \leq 10(i=4)$ respectively. From Table 1 , it is observed that $\mathrm{A}_{1}$ consists of ten attributes and $A_{2}$ consists of five attributes. Eleven attributes are considered as per $A_{3}$ and four as per $A_{4}$. The total ranges obtained by the individual four groups of the attributes corresponding to $\mathrm{A}_{1}, \mathrm{~A}_{2}, \mathrm{~A}_{3}$ and $\mathrm{A}_{4}$ are given in Table 2 .

Table 2. Total of attributes corresponding to $A_{1}, A_{2}, A_{3}$ and $A_{4}$ as per the divided four groups.

\begin{tabular}{|c|c|c|c|c|}
\hline $\begin{array}{c}\text { Summation of the attributes } \\
\text { corresponding to }\end{array}$ & Low & Medium & High & VHigh \\
\hline $\mathrm{A}_{1}$ & $0-25$ & $25-50$ & $50-75$ & $75-100$ \\
\hline $\mathrm{A}_{2}$ & $0-12.5$ & $12.5-25$ & $25-37.5$ & $37.5-50$ \\
\hline $\mathrm{A}_{3}$ & $0-27.5$ & $27.5-55$ & $55-82.5$ & $82.5-110$ \\
\hline $\mathrm{A}_{4}$ & $0-10$ & $10-20$ & $20-30$ & $30-40$ \\
\hline
\end{tabular}

As discussed above, the set of minimum scores assigned to each of the attributes according to the four groups are given as $Y_{i}=\{0,2.5,5$ and 7.5$\}$; where $i=1$ to 4 . An appropriate score assigned to the attribute based on its present value/condition is computed by using (1) $[28,29]$. It can exist in any of the four groups of that particular attribute.

$$
\text { Attribute score }=Y_{i}+\left[\left(\frac{x-g_{i}}{h_{i}-g_{i}}\right) \times 2.5\right]
$$

where, $x$ is the present value of the attribute/test, and $g_{i}$ and $h_{i}$ are the lower and upper limits of the four groups corresponding to that attribute. In this work, in total twenty-five transformers were tested and examined to validate the present approach. For the sake of brevity, the detailed analysis of five transformers has been presented in Tables 3 and 4 . However, final results of all the transformers are given in section 3 . 
Further, the scores obtained for attributes corresponding to $A_{1}, A_{2}, A_{3}$ and $A_{4}$ have been summarized [29]. These are denoted by $X_{1}, X_{2}, X_{3}$ and $X_{4}$. These summarized values can exist in any of the four corresponding groups given in Table 2 . These values can determine the overall health condition of the transformers. However, the health assessment of transformers based on these values (i.e. $X_{1}, X_{2}, X_{3}$ and $X_{4}$ ) is not reliable. It is very often observed that $X_{1}$ may lie in group 1 , whereas the other inputs lie in some other groups [29, 30]. In such cases it is difficult to determine the overall condition of a transformer. Consequently, it necessitates further investigations of these inputs in evaluating the accurate health condition of transformers. Therefore, a new fuzzy logic based health index model has been proposed in the present work to determine the overall health condition of transformers. The flow chart given in Figure 1 discusses the proposed methodology of the present work.

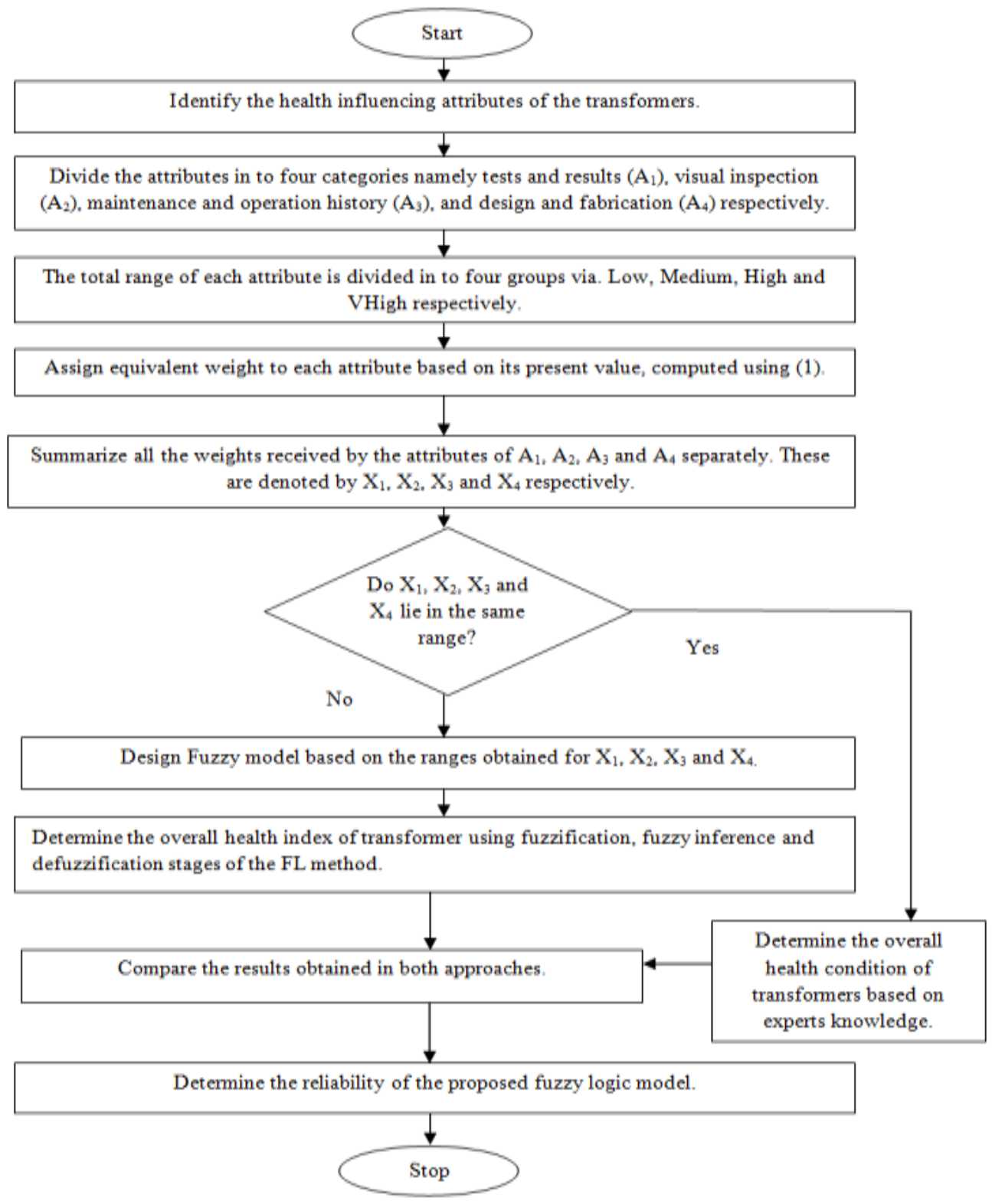


Figure 1 Proposed research methodology of the present work for an accurate decision making for transformers.

Table 3. Evaluation of five transformers rated $630 \mathrm{kVA}, 11 / 0.43 \mathrm{kV}$ operated by HPSEB, India.

\begin{tabular}{|c|c|c|c|c|c|c|c|c|c|c|c|}
\hline \multirow[t]{2}{*}{$\begin{array}{l}\mathrm{Sr} \\
\text { no } \\
\end{array}$} & Attribute & \multicolumn{2}{|c|}{ Transformer 1} & \multicolumn{2}{|c|}{ Transformer 2} & \multicolumn{2}{|c|}{ Transformer 3} & \multicolumn{2}{|c|}{ Transformer 4} & \multicolumn{2}{|c|}{ Transformer 5} \\
\hline & $\begin{array}{c}\text { Tests and } \\
\text { Measurements }\end{array}$ & Evaluation & Score & Evaluation & Score & Evaluation & Score & Evalution & Score & Evaluation & Score \\
\hline 1 & $\begin{array}{l}\text { DGA-TDCG } \\
(\mathrm{ppm})\end{array}$ & 3419 & 6.38 & 319 & 1.10 & 3419 & 6.38 & 5394 & 7.86 & 836 & 2.74 \\
\hline 2 & Oil BDV (kV) & 52 & 1.64 & 72 & 0.21 & 37 & 4 & 27 & 6.33 & 61 & 1.00 \\
\hline 3 & DP value (ppm) & 564 & 4.68 & 1261 & 0.86 & 357 & 6.19 & 192 & 7.60 & 1193 & 1.29 \\
\hline 4 & $\begin{array}{l}\text { Water content } \\
(\mathrm{ppm})\end{array}$ & 15 & 2.50 & 12 & 2 & 18 & 4 & 24 & 7.00 & 14 & 2.33 \\
\hline 5 & Furan content & 2.18 & 5.32 & 0.065 & 1.625 & 4.8 & 6.05 & 7 & 6.67 & 0.07 & 1.75 \\
\hline 6 & IFT $(\mathrm{mN} / \mathrm{m})$ & 18.6 & 4.30 & 18 & 4 & 17.59 & 3.79 & 26 & 7.60 & 16 & 3.00 \\
\hline 7 & Dissipation factor & 0.149 & 3.72 & 0.089 & 2.22 & 0.21 & 5.25 & 0.38 & 8.17 & 0.16 & 4.00 \\
\hline 8 & $\begin{array}{c}\text { Turns ratio (in \% } \\
\text { deviation) }\end{array}$ & 0.34 & 5.50 & 0.05 & 1.25 & 0.16 & 3.25 & 0.72 & 8.60 & 0.23 & 4.13 \\
\hline 9 & $\begin{array}{l}\text { Insulation } \\
\text { resistance (ohm) }\end{array}$ & 2819 & 3.40 & 4584 & 0.52 & 2379 & 5.60 & 1835 & 7.71 & 3294 & 2.13 \\
\hline 10 & $\begin{array}{l}\text { Acidity }(\mathrm{mg} \\
\mathrm{KOH} / \mathrm{g})\end{array}$ & 0.086 & 3.66 & 0.025 & 2.08 & 0.089 & 1.27 & 0.23 & 6.33 & 0.05 & 2.92 \\
\hline & $\begin{array}{l}\text { Total score of } \mathrm{A}_{1} \\
\text { (i.e. } \mathrm{X}_{1} \text { ) }\end{array}$ & & 41.13 & & 15.89 & & 45.8 & & 73.86 & & 25.29 \\
\hline
\end{tabular}

Table 4. Evaluation of five transformers rated $630 \mathrm{kVA}, 11 / 0.43 \mathrm{kV}$ operated by HPSEB, India.

\begin{tabular}{|c|c|c|c|c|c|c|c|c|c|c|c|}
\hline \multirow[t]{2}{*}{$\begin{array}{l}\mathrm{Sr} \\
\mathrm{No} \\
\end{array}$} & \multirow{2}{*}{ Attribute } & \multicolumn{2}{|c|}{ Transformer 1} & \multicolumn{2}{|c|}{ Transformer 2} & \multicolumn{2}{|c|}{ Transformer 3} & \multicolumn{2}{|c|}{ Transformer 4} & \multicolumn{2}{|c|}{ Transformer 5} \\
\hline & & Evaluation & Score & Evaluation & Score & Evaluation & Score & Evaluation & Score & Evaluation & Score \\
\hline & Visual Inspection & & & & & & & & & & \\
\hline 1 & $\begin{array}{l}\text { Presence of dust } \\
\text { and } \operatorname{dirt}(\mathrm{mm})\end{array}$ & little & 4 & 0 & 0 & Medium & 6 & medium & 7 & little & 3.00 \\
\hline 2 & Oil leakage & none & 0 & 0 & 0 & Little & 4 & little & 5 & none & 0.00 \\
\hline 3 & PD white powder & low & 3 & 0 & 0 & Low & 4 & medium & 7 & none & 0.00 \\
\hline 4 & Appearance of oil & BY & 3 & PYC & 1.5 & brown & 6 & brown & 7 & PYC & 2.00 \\
\hline \multirow[t]{3}{*}{5} & Core noise & 15.8 & 1.57 & 24.6 & 7.20 & 28.19 & 8.29 & 23.89 & 6.68 & 5.13 & 4.63 \\
\hline & $\begin{array}{l}\text { Total score of } \mathrm{A}_{2} \\
\text { (i.e. } \mathrm{X}_{2} \text { ) }\end{array}$ & & 11.57 & & 8.70 & & 28.29 & & 32.68 & & 9.63 \\
\hline & $\begin{array}{l}\text { Maintenance and } \\
\text { operation history }\end{array}$ & & & & & & & & & & \\
\hline 1 & Oil age (years) & 37 & 6.5 & 16 & 3.5 & 52 & 8.08 & 64 & 9.08 & 14 & 3.17 \\
\hline 2 & $\begin{array}{l}\text { Winding age } \\
\text { (years) }\end{array}$ & 37 & 6.5 & 16 & 3.5 & 52 & 8.08 & 64 & 9.08 & 14 & 3.17 \\
\hline 3 & Core age (years) & 37 & 6.5 & 16 & 3.5 & 52 & 8.08 & 64 & 9.08 & 14 & 3.17 \\
\hline 4 & Winding faults & twice & 6 & once & 4 & twice & 7 & $>$ twice & 9.00 & none & 0.00 \\
\hline 5 & Bushings faults & once & 4 & once & 4 & twice & 7 & $>$ twice & 9.00 & once & 4.00 \\
\hline 6 & $\begin{array}{l}\text { Bushing ambient } \\
\text { temperature }\end{array}$ & 49 & 4.75 & 47 & 4.25 & 59 & 7.25 & 53 & 5.75 & 42 & 3.00 \\
\hline 7 & Average load (\%) & 74 & 1.71 & 62.1 & 0.86 & 73 & 1.64 & 93 & 6.50 & 81 & 4.50 \\
\hline 8 & $\begin{array}{l}\text { Repairs occurred } \\
\text { in core }\end{array}$ & twice & 6 & 0 & 0 & Once & 4 & $>$ twice & 9.00 & none & 0.00 \\
\hline 9 & Power factor & 0.95 & 3.75 & 0.87 & 6.5 & 0.87 & 6.5 & 0.83 & 8.00 & 0.93 & 4.25 \\
\hline 10 & $\begin{array}{l}\text { Ambient } \\
\text { temperature }\left({ }^{0} \mathrm{C}\right)\end{array}$ & 34 & 1.16 & 34 & 1.16 & 34 & 1.16 & 34 & 1.17 & 34 & 1.17 \\
\hline \multirow[t]{3}{*}{11} & Tank damages & none & 0 & none & 0 & medium & 7 & high & 9.00 & none & 0.00 \\
\hline & $\begin{array}{l}\text { Total score of } \mathrm{A}_{3} \\
\text { (i.e. } \mathrm{X}_{3} \text { ) }\end{array}$ & & 46.88 & & 31.28 & & 102.4 & & 84.67 & & 26.42 \\
\hline & $\begin{array}{l}\text { Design and } \\
\text { Fabrication }\end{array}$ & & & & & & & & & & \\
\hline 1 & $\begin{array}{l}\text { Fire protection } \\
\text { system }\end{array}$ & MP & 3 & $\mathrm{AP}$ & 1 & MAP & 4 & DO & 9 & MP & 2.5 \\
\hline 2 & Type of cooling & FOFAC & 4 & WC & 1 & FOFAC & 4 & $\mathrm{ONC}$ & 9 & WC & 2.5 \\
\hline 3 & Type of radiator & tabular & 4 & flange & 1 & other & 7 & other & 7.5 & flange & 2 \\
\hline \multirow[t]{2}{*}{4} & Type of bushing & OIPB & 1 & OIPB & 1 & RIB & 7 & RBB & 9 & OIPB & 2 \\
\hline & $\begin{array}{l}\text { Total score of } \mathrm{A}_{4} \\
\text { (i.e. } \mathrm{X}_{4} \text { ) }\end{array}$ & & 12 & & 4 & & 22 & & 34.5 & & 9 \\
\hline
\end{tabular}




\section{The Proposed Fuzzy-Logic Health Index Model}

In the present work, the total scores obtained by the four categories $\mathrm{X}_{1}, \mathrm{X}_{2}, \mathrm{X}_{3}$ and $\mathrm{X}_{4}$ have been used as inputs for the proposed fuzzy model. Based on these four inputs, the overall health index assessment of transformers follows three different stages of fuzzy logic namely, fuzzification, fuzzy inference and defuzzification respectively. These stages are discussed in the following sub-sections.

\section{A. Fuzzification}

Fuzzification converts the precise inputs into fuzzy (i.e imprcise) by using the suitable membership functions (MFs) [31, 32]. A membership function (MF) is a curve that defines how a given input is mapped to a degree of membership (DOM), between 0 and 1 , of the fuzzy sets (imprecise sets) [33]. It has different shapes namely, triangular, trapezoidal, Gaussian and Gauss2. In the present work, a commonly utilized trapezoidal MF is used to represent the four inputs (i.e. from $X_{1}$ to $X_{4}$ ) of the proposed model. It is given by (2) [34].

$$
\text { Membership function }=\max \left\{\min \left(\frac{X-a}{c_{1}-a}, 1, \frac{b-X}{b-c_{2}}\right), 0\right\}
$$

where $X$ is an input. $a$ and $b$ are the lower and upper limits of the trapezoidal MF (Figure 2), and $c_{1}$ and $c_{2}$ are its centers [33]. Inputs between $c_{1}$ and $c_{2}$ of the MF attain a maximum DOM of unity. Whereas, the input between $a$ and $c_{1}$, or between $c_{2}$ and $b$, have a DOM less than unity. Thus, the fuzzification converts the inputs from precise to fuzzy (imprecise), between 0 and 1 .

In the present work, the inputs of the proposed model were fuzzified into four trapezoidal shaped MFs (fuzzy sets) namely Low, Medium, High and Very High (VHigh) respectively, as shown in Figure 2. The lower and upper limits for the MFs of each input were selected in accordance to Table 2 .

An overlap of 10 was maintained between the adjacent MFs of $X_{1}$ and $X_{3}$, whereas it was set as 5 for $X_{2}$ and $X_{4}$ adjacent MFs. At these values, the adjacent MFs of each input can achieve 50\% overlap, in accordance to [18, 32]. For each MF, $c_{1}$ and $c_{2}$ can be obtained from the overlaps between the adjacent MFs and the lower and upper limits [18]. The determination of the overall health condition of the transformers based on the designed fuzzy sets is more reliable than that based on precise sets [33].

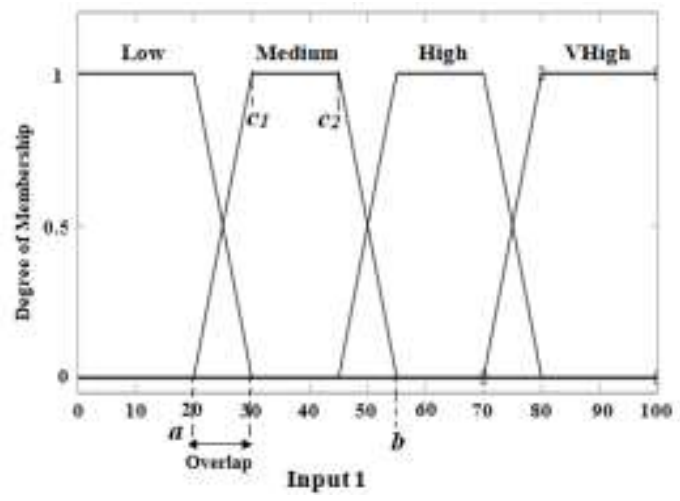

Figure 2. Membership functions and degrees of membership for input 1 i.e. $X_{1}$. 
Similarly, the plots were obtained for the MFs of remaining three inputs. The lower and the upper limits, and the centers for each of the four MFs for the inputs are given in Table 5.

Table 5. Lower and upper limits, and centers for all input MFs of the proposed FL model.

\begin{tabular}{|c|c|c|c|c|c|c|c|c|c|c|c|c|c|c|c|c|}
\hline \multirow{2}{*}{ Input } & \multicolumn{4}{|c|}{ Low } & \multicolumn{4}{|c|}{ Medium } & \multicolumn{4}{|c|}{ High } & \multicolumn{4}{|c|}{ VHigh } \\
\hline & $a$ & $c_{l}$ & $c_{2}$ & $b$ & $A$ & $c_{l}$ & $c_{2}$ & $b$ & $a$ & $c_{1}$ & $c_{2}$ & $b$ & $a$ & $c_{l}$ & $c_{2}$ & $b$ \\
\hline $\mathrm{X}_{1}$ & 0 & 0 & 20 & 30 & 20 & 30 & 45 & 55 & 45 & 55 & 70 & 80 & 70 & 80 & 100 & 100 \\
\hline $\mathrm{X}_{2}$ & 0 & 0 & 10 & 15 & 10 & 15 & 22.5 & 27.5 & 22.5 & 27.5 & 35 & 40 & 35 & 40 & 50 & 50 \\
\hline $\mathrm{X}_{3}$ & 0 & 0 & 22.5 & 32.5 & 22.5 & 32.5 & 50 & 60 & 50 & 60 & 77.5 & 87.5 & 77.5 & 87.5 & 110 & 110 \\
\hline $\mathrm{X}_{4}$ & 0 & 0 & 7.5 & 12.5 & 7.5 & 12.5 & 17.5 & 22.5 & 17.5 & 22.5 & 27.5 & 32.5 & 27.5 & 32.5 & 40 & 40 \\
\hline
\end{tabular}

The output of the proposed model (i.e. transformer overall health index) covers the range between 0 and 1. It is divided into four trapezoidal shaped MFs namely Excellent, Good, Poor and Worst, shown in Figure 3. The limits for these output MFs were selected as per [32]. An overlap of 0.1 was maintained between the adjacent MFs of the output, following the same $50 \%$ overlap between any two adjacent MFs (as in case of the input MFs). The transformers with an Excellent health index not require any remedial action, those with a Good index requires filtration of its oil, while that with a Poor health index requires reclamation. Transformers with Worst health index require immediate replacement [12, 13].

Figure 4 shows the schematic representation of the proposed FL based transformer overall health index model. The values for the inputs of the model correspond to Transformer 1 , detailed in Tables 3 and 4. Based on these inputs, the corresponding output i.e. overall health index (OHI) of the transformer obtained from the FL model is also seen in the figure.

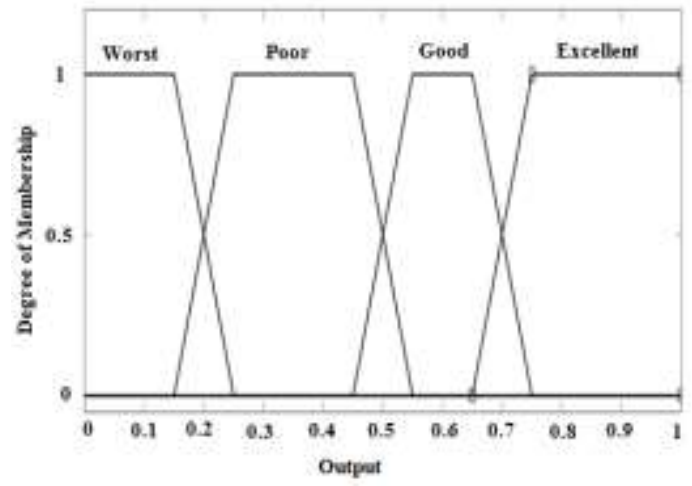

Figure 3. Output membership functions.

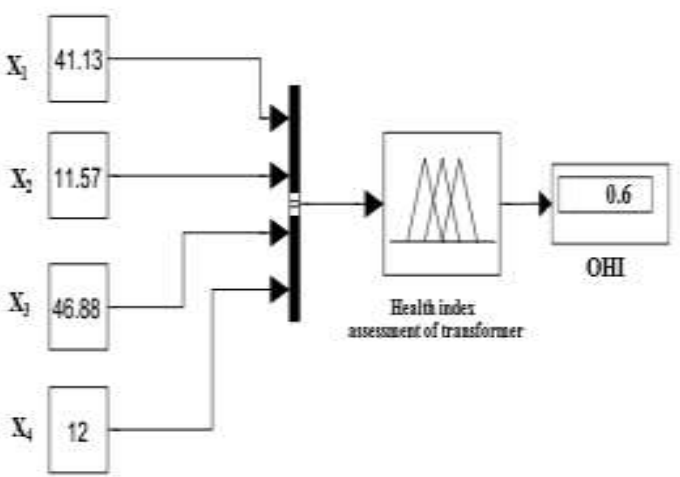

Figure 4. Proposed fuzzy logic model to determine the overall health index of transformers. 
In case of Transformer 1 , the total scores obtained by $X_{1}, X_{2}, X_{3}$ and $X_{4}$ are 41.13, $11.57,46.88$ and 12 respectively. $X_{1}=41.13$ corresponds to a DOM of 1 within the Medium MF, by using (2). DOMs of 1 within the Medium MF were also found for $\mathrm{X}_{3}$ and $\mathrm{X}_{4}$. However, in case of $\mathrm{X}_{2}$ with a value of 11.57 , there are two possible DOM values of 0.686 within the Excellent MF, and 0.314 within the Good MF respectively. Thus the precise inputs were converted into imprecise form. The corresponding output MFs based on these fuzzified inputs have been determined in fuzzy inference stage of the method.

\section{B. Fuzzy inference}

Fuzzy inference is the process of mapping the inputs with output by specifically designed rules [33]. In the present work, a widely used Mamdani maximum-minimum fuzzy inference method is used. This method determines the output MFs based on the set of fuzzified inputs and the designed fuzzy rules. Further it truncates the output MFs at their minimum DOM value [33].

In the present model, a total of forty specially designed fuzzy rules detailed in Figure 5 were generated based on the four inputs. Input 1 was given the highest priority in the design of the fuzzy rules. The reason is that the data corresponding to tests and measurements of transformers consist of very pertinent information regarding the health condition. Subsequently, input $X_{3}$ and $X_{2}$ were given priority next to input 1 . For every condition of $X_{1}$ (from Low to VHigh) there are many possible combinations from the other three inputs. Thus, for each of the four conditions for $\mathrm{X}_{1}$, the most commonly identified combinations of the other three inputs were adopted. For instance, for a Low range of $\mathrm{X}_{1}$, the most commonly identified situations of the other three inputs are detailed in Table 6.

Table 6. Fuzzy rules generated between the Low range of $X_{1}$ and the commonly identified combinations of the other three inputs.

\begin{tabular}{|c|c|c|c|c|}
\hline IF Input 1 is & AND Input 2 is & AND Input 3 is & AND Input 4 is & THEN Output is \\
\hline Low & Low & Low & Low & Excellent \\
\hline Low & Medium & Medium & Medium & Good \\
\hline Low & High & High & High & Poor \\
\hline Low & Vhigh & Vhigh & Vhigh & Worst \\
\hline
\end{tabular}

The designed fuzzy rules numbered from 1 to 4 in Figure 5 were formed accordingly (i.e. as per Table 6). Similarly, the remaining fuzzy rules were generated in such a way so as to cover all the possible health conditions of the transformer. The representation of various shapes seen in Figure 5 is detailed in Table 7.

Table 7. Representation of various shapes seen in Figure 5.

\begin{tabular}{|l|l|}
\hline Shape & Indication \\
\hline$\square:$ Black coloured unfilled shape & Input MF considered in the designed fuzzy rule \\
\hline$\square:$ Yellow filled shape & Corresponding input MF as per the given input \\
\hline$\square$ : Blue coloured unfilled shape & Output MF for the specified set of inputs \\
\hline$\square$ : Blue filled shape & $\begin{array}{l}\text { Corresponding output MF as per the given set of } \\
\text { inputs }\end{array}$ \\
\hline
\end{tabular}




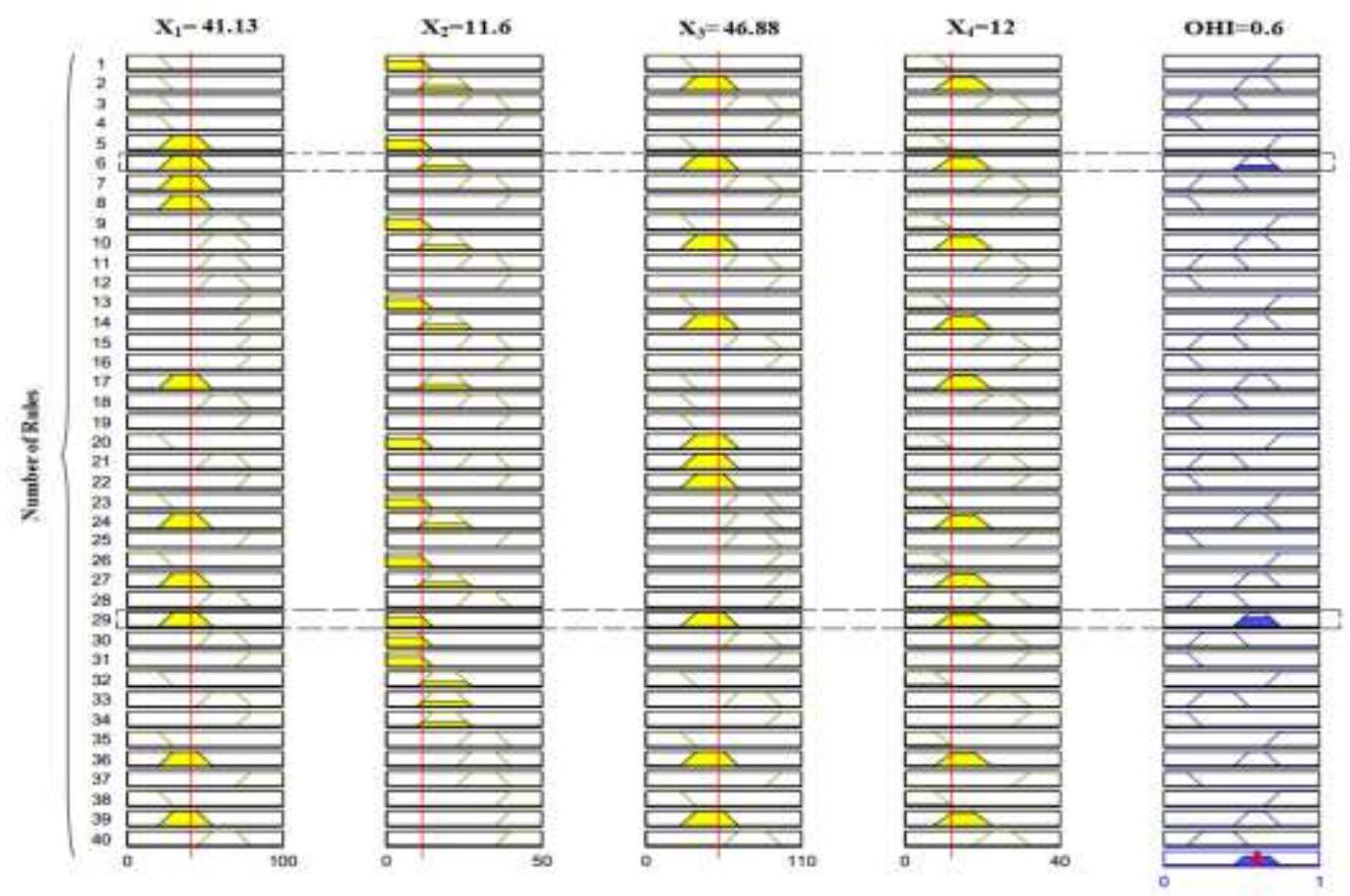

Figure 5. Graphical representation of the devSeloped 40 fuzzy rules to determine the transformer overall health index.

The red-coloured vertical lines in Figure 5 indicate the four inputs corresponding to Transformer 1. The limits of all unfilled shapes as well as darkly filled areas specify the lower and the upper limits of the MFs in the designed fuzzy rules. The interpretation of four out of the forty rules in Figure 5 is given below to aid understanding of the rules.

Rule 1: IF (each of the four inputs is Low) THEN (the overall health condition of the transformer is Excellent).

Rule 6: IF (each of the four inputs is Medium) THEN (the overall health condition of the transformer is Good).

Rule 29: IF (input2 is Low) AND (each of the other three inputs is Medium) THEN (the health condition of the transformer is Good).

Rule 40: IF (input2 is VHigh) AND (each of the other three inputs is High) THEN (the health condition of the transformer is Poor).

In the present work, the Mamdani maximum-minimum fuzzy inference method is used to determine the output MFs from the given set of inputs. It truncates the output MFs at their minimum DOM values. Such a truncation is shown in Figure 6 in relation to executed Rules 6 and 29, applied to Transformer 1. The input MFs are shown in the first four boxes, and the output MF is shown by the blue coloured box. The output MFs as per inputs $X_{1}, X_{3}$ and $X_{4}$ for this test case were Good with a DOM of 1 , but for input $\mathrm{X}_{2}$ the output MF was Good having DOMs of 0.314 and 0.686 . The fuzzy inference method therefore truncates the output MFs at the lower DOM values i.e 0.314 and 0.686 respectively. Consequently, the output MF according to the remaining thirty-eight fuzzy rules depicted in Figure 5 was zero, for the same set of inputs of Transformer 1. The reason is that one or more inputs in this test case did not lie within the ranges specified in these thirty eight fuzzy rules. The final precise output from the truncated output MF is determined using the defuzzification stage of the model. 

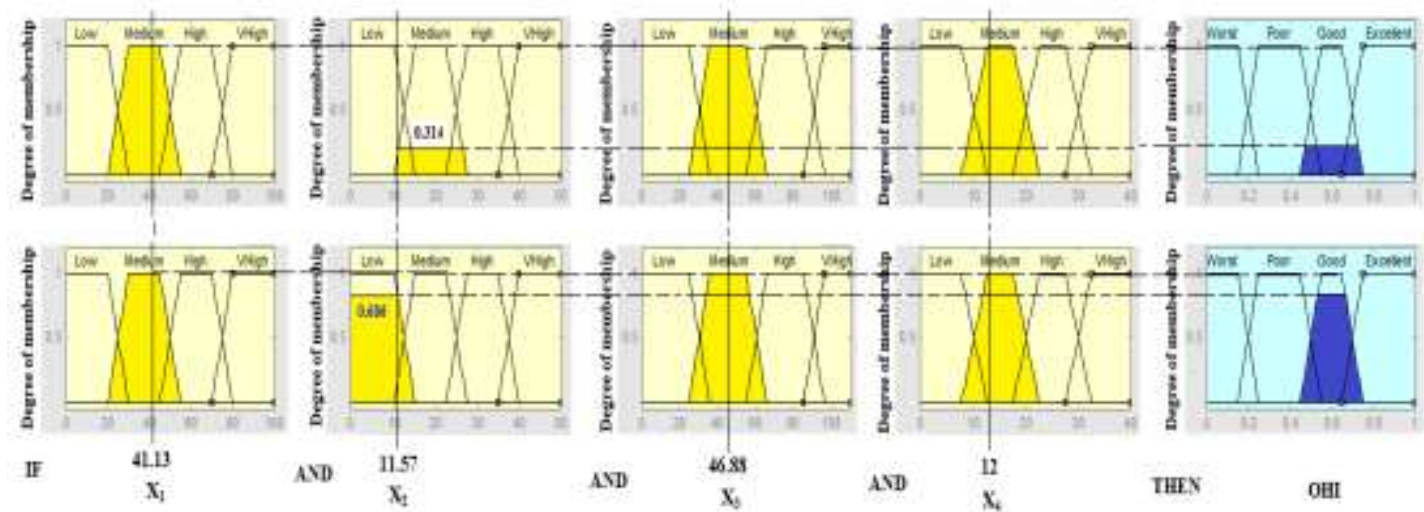

Figure 6. Pictorial representation of truncated output membership functions (in blue) for the overall health index of transformer 1, according to rules 6 and 29.

\section{Defuzzification}

A precise quantitative value from the truncated output MFs is determined in defuzzification stage of the method. In the present work, defuzzification was performed by the most popular center of gravity method. It determines the center of gravity or the centroid ( $Z_{0}$ ) of the area bounded by the truncated output MFs [33, 34]. It is obtained by

$$
Z_{0}=\frac{\int z \cdot \mu(z) d z}{\int \mu(z) d z}
$$

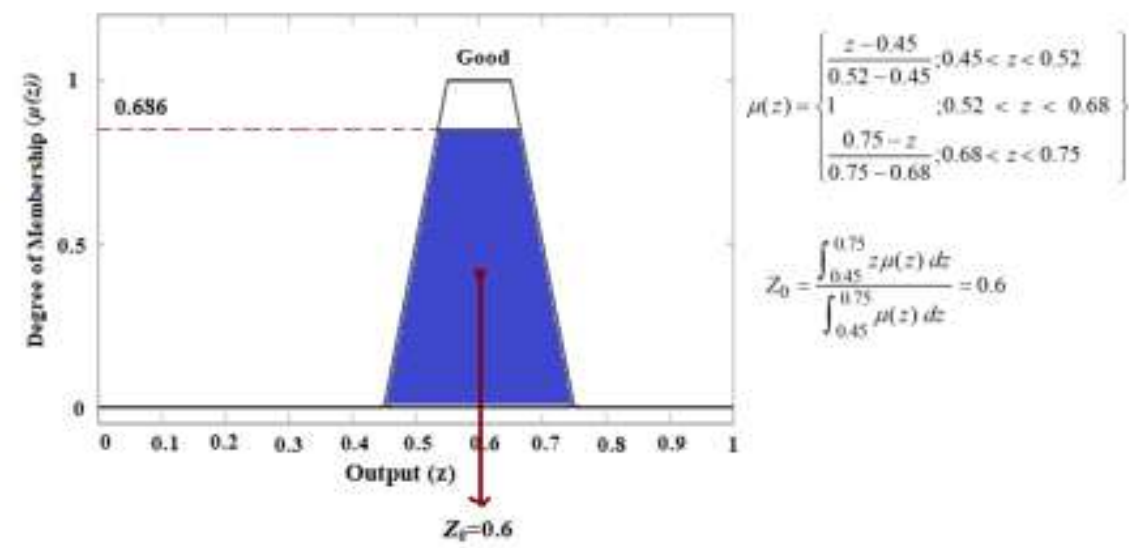

Figure 7. Defuzzification of the truncated output MF using the centre of gravity method.

where $z$ is the output variable and $\mu(z)$ is the DOM of the truncated output MF. For Transformer 1, the given set of inputs was truncated the Good output MFs. Its centroid which is the quantitative overall health index (OHI) of transformers was obtained as 0.6 (Figure 7). This value indicates that the health condition of Transformer 1 is Good. However the transformer requires continuous supervision and maintenance for its better performance [35]. Similarly, the health indexes for the remaining transformers were determined by the proposed FL method, and given in Table 8. The corresponding health conditions are also indicated in the table. Furthermore, the health conditions of transformers based on different tests and measurements (mainly DGA, BDV, DP, FP and IFT) and the experience of transformer diagnostic experts as well as field engineers [24-27] are given in column 8 of the table. The 
MVA ratings of the transformers are in the range 5-50 MVA, and their $\mathrm{kV}$ ratings are in the range $6-220 \mathrm{kV}$. These transformers are operated by HPSEB, India.

From Table 8, it is observed using the proposed FL method that the overall health condition of transformers 2, 5, 7, 9, 12, 17, 20, 21 and 25 is Excellent. Whereas, the health condition for transformers 1, 6, 8, 10, 15, 22 and 24 is Good, and it is Poor for transformers 3, 16, 18, and 23. Similarly, the health condition of the transformers $4,11,13,14$ and 19 is Worst. The health conditions obtained for these twenty-five transformers based on the various conventional test results and the experience of transformer diagnostic experts [24-27] can also be observed from the table. However, these results are different in case of transformers $3,8,11,12,13,18,19$, 21 and 23. The reason for this is that the diagnostic experts evaluate the health condition of the transformers based on a fewer number of the test results. Whereas the proposed FL method depends on a combined influence of all the factors of transformers including several test results.

For instance, in case of Transformer 3, the overall health condition based on tests data and experts knowledge was Good. But this output is not reliable and accurate. This is because some test results of the transformer exist in Good range of health condition (Tables 3 and 4), and a few of them lie in Poor range. Therefore, an accurate health assessment of the transformer is not achievable by this approach. Consequently, these shortcomings have been successfully overcome by the proposed FL method. For the same test transformer, the overall health index based on all the influencing factors was obtained as 0.35 , using the proposed FL method. It indicates a Poor health condition of the transformer. This output is different from the conventional method output. This is due to the reason that the total scores gained by the attributes corresponding to $\mathrm{A}_{2}, \mathrm{~A}_{3}$ and $\mathrm{A}_{4}$ are high. Also the transformer had been subjected to various types of severe faults at several times and hence the degradation rate of the insulation is high. Therefore, this transformer requires reclamation to improve its operating performance. Thus, the proposed FL model accurately determines the overall health index of transformers based on several influencing factors.

Table 8- The output obtained from the proposed FL model.

\begin{tabular}{|c|c|c|c|c|c|c|c|c|c|}
\hline \multirow{2}{*}{$\begin{array}{c}\text { Transformer } \\
\text { No. }\end{array}$} & \multicolumn{4}{|c|}{$\begin{array}{l}\text { Total scores obtained by } X_{1}, X_{2}, X_{3} \\
\text { and } X_{4}\end{array}$} & \multirow{2}{*}{$\begin{array}{l}\text { Output of } \\
\text { the } \\
\text { proposed } \\
\text { FL method } \\
\text { (i.e. OHI) }\end{array}$} & \multirow{2}{*}{$\begin{array}{l}\text { Transformer } \\
\text { health } \\
\text { condition } \\
\text { obtained } \\
\text { from the } \\
\text { proposed FL } \\
\text { method }\end{array}$} & \multirow{2}{*}{$\begin{array}{l}\text { Output } \\
\text { of the } \\
\text { method } \\
\text { used in } \\
\text { [32] }\end{array}$} & \multirow{2}{*}{$\begin{array}{c}\text { Transformer } \\
\text { health } \\
\text { condition } \\
\text { obtained from } \\
\text { the method in } \\
{[32]}\end{array}$} & \multirow{2}{*}{$\begin{array}{c}\text { Transformer } \\
\text { health } \\
\text { condition } \\
\text { based on the } \\
\text { diagnostic } \\
\text { experts [24-27] }\end{array}$} \\
\hline & $\mathrm{X}_{1}$ & $\mathrm{X}_{2}$ & $\mathrm{X}_{3}$ & $\mathrm{X}_{4}$ & & & & & \\
\hline 1 & 41.13 & 11.57 & 46.88 & 12 & 0.60 & Good & 0.56 & Good & Good \\
\hline 2 & 15.89 & 8.70 & 31.28 & 4 & 0.84 & Excellent & 0.89 & Excellent & Excellent \\
\hline 3 & 45.80 & 28.29 & 102.40 & 22 & 0.35 & Poor & 0.28 & Poor & Good \\
\hline 4 & 73.86 & 32.68 & 84.67 & 34.50 & 0.11 & Worst & 0.41 & Poor & Worst \\
\hline 5 & 25.31 & 9.63 & 26.41 & 9 & 0.77 & Excellent & 0.79 & Excellent & Excellent \\
\hline 6 & 27.81 & 17.97 & 29.50 & 11.38 & 0.67 & Good & 0.71 & Good & Good \\
\hline 7 & 22.19 & 7.03 & 22.05 & 10.88 & 0.83 & Excellent & 0.89 & Excellent & Excellent \\
\hline 8 & 50.31 & 24.53 & 51.24 & 19.38 & 0.47 & Good & 0.43 & Good & Poor \\
\hline 9 & 8.43 & 7.03 & 13.35 & 0.37 & 0.85 & Excellent & 0.89 & Excellent & Excellent \\
\hline 10 & 72.81 & 30.78 & 19.57 & 7.62 & 0.50 & Good & 0.58 & Good & Good \\
\hline 11 & 91.56 & 43.28 & 90.37 & 35.38 & 0.19 & Worst & 0.21 & Worst & Poor \\
\hline 12 & 49.06 & 11.09 & 27.02 & 8.62 & 0.78 & Excellent & 0.74 & Excellent & Good \\
\hline 13 & 72.19 & 38.59 & 84.16 & 30.63 & 0.22 & Worst & 0.29 & Worst & Poor \\
\hline 14 & 59.69 & 35.78 & 782.57 & 34.13 & 0.12 & Worst & 0.19 & Worst & Worst \\
\hline 15 & 27.19 & 21.09 & 42.55 & 8.62 & 0.62 & Good & 0.71 & Good & Good \\
\hline 16 & 63.44 & 37.03 & 68.01 & 29.13 & 0.35 & Poor & 0.30 & Poor & Poor \\
\hline 17 & 25.94 & 9.53 & 26.40 & 9.87 & 0.75 & Excellent & 0.71 & Excellent & Excellent \\
\hline 18 & 44.69 & 37.03 & 56.21 & 19.63 & 0.44 & Poor & 0.39 & Poor & Good \\
\hline 19 & 78.44 & 44.84 & 83.54 & 31.13 & 0.18 & Worst & 0.11 & Worst & Poor \\
\hline 20 & 27.19 & 11.09 & 30.75 & 6.87 & 0.83 & Excellent & 0.89 & Excellent & Excellent \\
\hline 21 & 27.19 & 13.28 & 28.26 & 9.62 & 0.72 & Excellent & 0.72 & Excellent & Good \\
\hline 22 & 47.81 & 23.59 & 49.38 & 21.63 & 0.43 & Good & 0.48 & Good & Good \\
\hline 23 & 69.06 & 36.41 & 79.81 & 30.88 & 0.28 & Poor & 0.21 & Poor & Worst \\
\hline 24 & 72.81 & 23.59 & 51.24 & 19.88 & 0.51 & Good & 0.58 & Good & Good \\
\hline 25 & 6.56 & 7.34 & 25.78 & 10.38 & 0.83 & Excellent & 0.82 & Excellent & Excellent \\
\hline
\end{tabular}


Further, the results of the present method 3 have been compared with those determined in accordance to the method proposed in [32]. The FL model in [32] relies upon six inputs viz. water content, acidity, BDV, DF, 2-FAL and TDCG (Table 3), and a reduced set of thirty-three specially designed fuzzy rules. However, six inputs each with more than three MFs generate a large number of possible fuzzy rules. Consequently, a reduced set of fuzzy rules may not incorporate the impact of all the considered inputs, thus may result sometimes in an inaccurate output health index for the transformers. Also the specially designed fuzzy rules may vary from expert to expert. Such shortcomings of previously proposed fuzzy logic models have been successfully overcome in the present work by integrating the multi-criterion analysis with the proposed FL method.

Comparative analysis of both the methods (i.e. the proposed and that given in [32]) shows that the health conditions of 24 transformers out of 25 are similar as summarized in Table 8 . It indicates that $96 \%$ of the results of both the methods are identical. However, for transformer 4 (Tables 3 and 4) health condition obtained is Worst, using the present proposed model, whereas, for this transformer, Poor health condition has been obtained using the method given in [32]. For Transformer $4, X_{1}=73.86, X_{2}=32.68, X_{3}=84.67$ and $X_{4}=34.5$, it is observed from Table 2 that the values of $X_{1}$ to $X_{4}$ exist in their respective VHigh range thereby, resulting in Worst output health condition of transformer. Thus, the proposed multi-criterion, multiattribute based FL model accurately determines the health index of the transformer. The last column of Table 8 presents the transformer health conditions based upon the opinion of the diagnostic experts [24-27]. The results obtained by the proposed method are quite comparable to the views of the field experts. These further validate the efficacy and efficiency of the proposed method. Based on the health conditions of the transformers anticipated timely remedial actions can be initiated so as to improve their operating performance and operating life span.

\section{Conclusion}

The present paper proposes a new fuzzy logic model to determine the overall health index of transformers. It incorporates the data obtained from several influencing factors of transformers which are categorized in to four attributes namely, tests and measurements, visual inspection, maintenance and operation history, and design and fabrication. Each attribute is divided in to four groups viz. low, medium, high and very high. Based on the ranges attained by the four categories, the fuzzy logic model has been designed to find the overall health index. The proposed methodology is very accurate, reliable and overcomes the shortcomings of the power transformer conventional health assessment methods which are normally based on fewer of the diagnostic tests results. The proposed method also suggests remedial actions to be initiated to improve the health index of the transformer.

\section{Acknowledgement}

Authors would like to thank the authorities of TEQIP-II of NIT Hamirpur India for providing the financial support with grant number NIT/HMR/TEQIP-II/Research \& Develpoment-19/2015/2157-63. Authors are also thankful to the Himachal Pradesh State Electricity Board (HPSEB)-India for providing the transformer oil samples, and the authorities of TIFAC-CORE Centre of NIT Hamirpur India for providing the necessary facilities to perform the experiments of the present research.

\section{References}

[1]. Bakar N A, Siada A A, A novel method of measuring transformer oil interfacial tension using UV-Vis spectroscopy, IEEE Electr Insul Mag, 32 (2016) 1-7.

[2]. Rahmati A, Sanaye P M, Protection of power transformer using multi criteria decisionmaking, Int J Electr Power and Energy Syst, 68 (2015) 294-303.

[3]. Ghoneima S S M, Tahab I B M, A new approach of DGA interpretation technique for transformer fault diagnosis, Int J Electr Power and Energy Syst, 81(2016) 265-274. 
[4]. Anjali C, Partha B, Roy N K, Kumbhakar P, Usage of nanotechnology based gas sensor for health assessment and maintenance of transformers by DGA method, Int $J$ Electr Power and Energy Syst, 45 (2013) 137-141.

[5]. Elanien A, Salama M M A, Asset management techniques for transformers, Electr Power Syst Research, 80 (2010) 456-464.

[6]. Arshad M, Islam S M, Khaliq A, Fuzzy Logic approach in power transformers management and decision making, IEEE Trans Dielectr Electr Insul, 21 (2014) 2343 2354.

[7]. Rogers R, IEEE and IEC codes to interpret incipient faults in transformer using gas in oil analysis, IEEE Trans Dielectr Electr Insul, 13 (1978) 349-354.

[8]. Arakelian V G, Effective diagnostics for oil-filled equipment, IEEE Electr Insul Mag, 18 (2002) 26-38.

[9]. Suna H C, Huanga Y C, Huang C M, A review of dissolved gas analysis in power transformers, Energy Procedia, 14 (2002) 1220-1225.

[10]. Duval M, New techniques for dissolved gas-in-oil analysis, IEEE Electr Insul Mag, 19 (2003) 6-15.

[11]. Muhamad N A, Phung B T, Blackburn T R, Lai K X, Comparative study and analysis of DGA methods for transformer mineral oil using fuzzy logic, IEEE Conference on Power Eng, (2007) 1301-1306.

[12]. IEEE guide for the interpretation of gases generated in oil-immersed transformers, IEEE Std C57 104-2008 (Revision of IEEE std C57 104-1991), 2009 C1-28.

[13]. IEEE guide for failure investigation, documentation and analysis for power transformers and shunt reactors. IEEE Std Board C57 125-2014 (Revision of IEEE Std C57 125-1991) 2014.

[14]. Lorin P, Transformer condition assessment: Methodologies and on-site repair solutions, IEEE Ind Appl Mag, 20 (2014) 50-57.

[15]. Faria H D, A review of monitoring methods for predictive maintenance of electric power transformers based on dissolved gas analysis, Rene and Sust Ener Rev, 46 (2015) 201209.

[16]. Deepika B, Raj Kumar B, Hari Om G, Function analysis based rule extraction from artificial neural networks for transformer incipient fault diagnosis, Int J Electr Power and Energy Syst, 43 (2012) 1196-1203.

[17]. Siada A, Hmood S, A new fuzzy logic approach to identify power transformer criticality using dissolved gas-in-oil analysis, Int Jour Electr Power and Energy Systems, 67 (2015) 401-408.

[18]. Siada A, Arshad M, Islam S, Fuzzy logic approach to identify transformer criticality using dissolved gas analysis, IEEE Power and Energy Society General Meeting, (2010) $1-5$.

[19]. Siada A, Correlation of furan concentration and spectral response of transformer oil using expert systems, IET Sci Measurement and Techn, 5 (2011) 183-188.

[20]. Khan S A, Equbal M D, Islam T, A comprehensive comparative study of DGA based transformer fault diagnosis using fuzzy logic and ANFIS models, IEEE Trans Dielectr Electr Insul, 22 (2015) 590-596.

[21]. Yang X, Nielsen S, Ledwich G, Investigations of dielectric monitoring on an energized transformer oil paper insulation system, IET Sci Measurement and Techn 9 (2015): 102112.

[22]. Eslamiana M, Vahidia B, Eslamian A, Thermal analysis of cast-resin dry-type transformers, Energy Convers and Management, 52 (2011) 2479-2488.

[23]. Zeleni M, Multiple criterion decision making, Tata Mc Graw-Hill, Delhi, India.

[24]. ABB Management Service Ltd., ABB Service Handbook for Transformers, 2007 Switzerland.

[25]. IEC 60422, Mineral insulating oils in electrical equipment-Supervision and maintenance guidance $2005,3^{\text {rd }}$ ed. 
[26]. Bharath heavy electrical limited, Transformers, $2^{\text {nd }}$ edition, Tata Mc-Graw Hill Education 2003, Delhi, India.

[27]. Indrajit Dasgupta, Design of transformers, Tata Mc-Graw Hill Education 2002, Delhi, India.

[28]. Stewart T J, Scenario analysis and multi-criteria decision making, Springer, Berlin.

[29]. Rahi O P, Chandel A K, Sharma M G, Refurbishment and up rating of old hydro power plants, Ind Jour of Pow and River Valley Devel, 2 (2010) 143-147.

[30]. Su Q, Mi C, Lai L L, Austin P, A fuzzy dissolved gas analysis method for diagnosis of multiple incipient faults in a transformer, IEEE Trans Power Syts, 15 (2000) 593-598.

[31]. Malika H, Kumar Yadav A, Mishraa S, Mehta T, Application of neuro-fuzzy scheme to investigate the winding insulation paper deterioration in oil-immersed power transformer, Int J Electr Power and Energy Syst 53 (2013) 256-271.

[32]. Elanien A, Salama M M M, Calculation of a health index for oil-immersed transformers rated under 69kV using fuzzy logic, IEEE Trans Dielectr Electr Insul, 27 (2012) 2029_ 2036.

[33]. Nedjah N, Mourelle L M, Fuzzy systems engineering theory and practice, Springer, 2005.

[34]. Karray F O, De Silva C W, Soft computing and intelligent systems design: Theory, tools and applications, Pearson/Addison-Wesley, 2004.

[35]. Jahromi A N, Piercy R, Cress S, Fan W, An approach to power transformer asset management using health index, IEEE Electr Insul Mag, 25 (2009) 20-34.
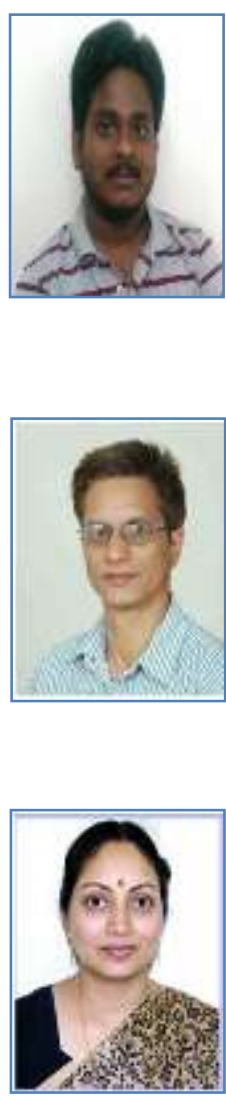

Chilaka Ranga (S'16) received the B. Tech. degree in Electrical and Electronics Engineering from Bapatla Engineering College, Bapatla (AP), India in 2010. He received his M.Tech. degree from National Institute of Technology, Hamirpur (HP), India, in 2012. Presently he is pursuing his $\mathrm{Ph} . \mathrm{D}$. from Department of Electrical Engineering, National Institute of Technology, Hamirpur (HP). His areas of interest are performance evaluation and health assessment of power transformers.

Ashwani Kumar Chandel (S'05-M'15) received his Ph.D. degree from Indian Institute of Technology Roorkee, India in 2005. Dr. Chandel joined the Department of Electrical Engineering, National Institute of Technology, Hamirpur, HP, India, as Lecturer in 1991, where presently he is working as a Professor. His research areas are harmonic estimation and elimination, condition monitoring of transformers. He is a Fellow of IETE, Member IEEE and Life Member of ISTE.

Rajeevan Chandel (S'05-M'15) received M.Tech. degree in Integrated Electronics from IIT Delhi, India, in 1997 and Ph.D. degree from IIT Roorkee in 2005. She joined as a Lecturer in E\&CED, NIT Hamirpur in 1990, where she is currently a Professor and is Dean (R\&C). She has five sponsored projects to her credit and over 50 research papers in journals of repute. Her research interests include electronics circuit modeling and lowpower design. Dr. Chandel is a Fellow of IETE(I), Life Member of ISTE(I) and Member IEEE. 